\title{
40
}

\section{Analysis of the screening formalisms in solar and stellar conditions}

H. DZITKO AND S. TURCK-CHIEZE

Service d'Astrophysique, DAPNIA. C.E. Saclay, 91191 Gif sur Yvette. France

P. DELBOURGO-SALVADOR AND CH. LAGRANGE

Service PTN. C.E. Bruyères le Châtel, BP 12, 91680 Bruyères le Châtel. France

\begin{abstract}
We discuss the quality of the electronic screening prescriptions usable in stellar evolution codes during hydrogen burning. The assumption $\log f=$ $H(0) / k T$ is compared to a precise formalism where the radial dependence of the screened potential of the 2 ions is introduced.
\end{abstract}

\subsection{The neutrino puzzle and the solar model accuracy.}

The microscopic description of the solar interior is crucial for both neutrino and acoustic mode frequency predictions, as that was pointed out by Turck-Chièze and Lopes (1993, TCL93). In this way, the best input physics have been looked for: precise composition, detailed opacity coefficients, and best nuclear reaction rates (see review of Turck-Chièze et al). As some neutrino sources are strongly dependent on the temperature and not really constraint by the luminosity $\left(\Phi_{\nu}\left({ }^{8} B\right) \propto T^{\mathbf{1 8}}\right)$, a good microscopic description is required (see table below). At present, the area between the nuclear and convective zones is rather under control by the determination of the sound speed behavior, but the nuclear region is not as well constraint by helioseismology, and the presence of mixing or of a small convective core is not excluded (Dzitko and Turck-Chièze, 1993). We have also noticed that the uncertainties on the nuclear reaction rates were still important (TCL93), and it is the reason why we consider here the screening effect produced by plasma electrons on the nuclear reaction rates. 
Table 40.1. Comparison of experimental results (in $S N U$ units $10^{-36}$ captures/atom/s) with Saclay neutrino predictions(TCL93).

\begin{tabular}{lll}
\hline \hline experimental results & Predictions & ratio $\frac{\exp }{t h}$ \\
\hline Chlorine experiment: & & \\
$2.33 \pm 0.25$ & $6.4 \pm 1.4$ & $0.36 \pm 0.04 \pm 0.08$ \\
Kamiokande experiment: & & \\
KII 0.28 \pm 0.03 evts/d & $0.48 \pm 0.12$ & $0.58 \pm 0.062 \pm 0.15$ \\
KIII & & $0.72 \pm 0.15 \pm 0.17$ \\
Gallium experiments: & $123 \pm 7$ & $0.47_{-0.22}^{+0.18} \pm 0.03$ \\
SAGE 58 $77 \pm 14$ & & $0.68 \pm 0.17 \pm 0.04$ \\
GALLEX I $83 \pm 19 \pm 8$ & & $0.79 \pm 0.19 \pm 0.05$ \\
GALLEX II $97 \pm 19 \pm 8$ & & \\
\hline \hline
\end{tabular}

\subsection{Screening effect in nuclear reactions}

In the central region of stars, most of the atoms are entirely striped of their atomic electrons. The nuclei are immersed in a sea of free electrons which cluster in their vicinity, lowering the repulsive Coulomb barrier, so that the probability of wave functions to tunnel through this barrier is increased. Thus, the nuclear reaction rates are enhanced over their vacuum values by the so called screening factor $f=\langle\sigma v\rangle_{\text {in plasma }} /\langle\sigma v\rangle_{\text {vacuum }}$ (1).

\subsubsection{Analytic form of the screening factor.}

The screening factor $f$ can be expressed as $f=\exp [H(0) / k T]$ if the internuclear spacing is much larger than the classical turning point. For stellar evolution applications, the analytic forms of the screening factors which have generally been used are those developed by Salpeter (1954, hereafter S54) and Graboske et al (1973, GWCD), namely:

$$
f_{w}=\exp \Lambda=\exp \left(Z_{1} Z_{2} e^{2}\right) /\left(R_{D} k T\right), R_{D}=\sqrt{k T /\left(4 \pi e^{2} n_{I} \xi^{2}\right)}
$$

$R_{D}$ is the Debye-Huckel radius, $\xi=\sqrt{\sum_{i}\left(Z_{i}\left(Z_{i}+\theta_{e}\right)\right) X_{i} / A_{i}}$ the rms charge of the plasma and $\theta_{c}$ the electronic degeneracy factor. $\Lambda$ is the natural screening parameter, ie the ratio of the Coulomb energy interaction to the kinetic energy of the reacting particles. $f_{u}$ is only valid when $\Lambda \ll 1$, that is, in the weak screening (WS) regime. Later GWGC presented a formalism recovering the limits of WS and SS (strong screening) regimes:

$$
\log f_{G}=k_{b} \eta_{b} \Lambda_{0}^{b}\left[\left(Z_{1}+Z_{2}\right)^{1+b}-Z_{1}^{1+b}-Z_{2}^{1+b}\right], \Lambda_{0}=1.8810^{8}\left(\rho / \mu T^{3}\right)^{\frac{1}{2}}
$$


$\Lambda_{0}$ is a charge independent variable characterizing the plasma. b lies between 1 and $2 / 3$ for WS or SS and 0.860 for IS (intermediate screening), value obtained with the cluster expansion theory. $\eta_{b}$ depends on the appropriate charge plasma average $\xi$. For WS $\eta_{b}=\xi, k_{b}=1 / 2$ leading to the classical Salpeter's formula. Thus, 3 screening regimes are defined: $\Lambda<0.1 \rightarrow$ WS, $0.1<\Lambda<5 \rightarrow$ IS, and $5<\Lambda \rightarrow$ SS.

In the previous standard assumptions, it was written $\log f=H(0) / k T=$ $\Delta U / k T$ where $\Delta U$ is the electrostatic energy difference of the 2 reacting nuclei between infinite separation and fusion, but Mitler showed that $\Delta F$, the difference in Helmholtz free energy, is better adapted than $\Delta U$ (Mitler 1977, hereafter M77). In fact, the first two terms of the expansion of the effective interaction energy are:

$$
U(r)=\frac{Z_{1} Z_{2} e^{2}}{r}-\frac{\Delta F(0)}{k_{B} T}, \quad \text { hence } \quad \log f_{M 0}=\frac{|\Delta F(0)|}{k T}
$$

This calculation is carried out in the 2 fluid approximation, within the statistical equilibrium assumption, and with a better charge density where 2 spherical domains around the reacting ions are defined and limited by some radius $r_{1}$ (roughly, the radius of the Wigner-Seitz sphere): if $r<$ $r_{1}$ the electron density is taken uniform (as in S54), and if $r>r_{1}$ the density distribution goes like $e^{-\kappa r} / r$, as in the Debye theory. This leads to a prescription which recovers the previous S54 and GWGC results in WS, and which is also valid for all regimes, but without any discontinuities over the $\Lambda$ range, as it is the case for the GWGC formalism (see figure 1.1).

\subsubsection{Numerical computation with radial dependence of the screened potential function $H(r)$}

In this case, one checks the validity of the expression (1). $H(0)$ is then replaced by $H(r)$ which accounts for the distorted ion-electron distribution around the 2 ions in interaction. At short distance, their common cloud is assumed to be uniformly distributed following an ellipsoid of revolution, and at large distance $H(r)$ turns into an usual Yukawa potential. This radial dependence involves a recalculation of the screened reaction rates $\langle\sigma v\rangle_{\text {screened }}$. The enhancement factor $f$ is thereby deduced as follows: $f_{M r}=\langle\sigma v\rangle_{\text {screened }} /\langle\sigma v\rangle_{\text {vacuum }}$.

\subsubsection{Accuracy of the screening formalisms.}

The analytic screening prescriptions were studied for the $p+{ }^{7} \mathrm{Be}$ and $p+{ }^{14} \mathrm{~N}$ reactions in the solar and stellar cases $\left(0.6<M<20 M_{\odot}\right)$ and compared 


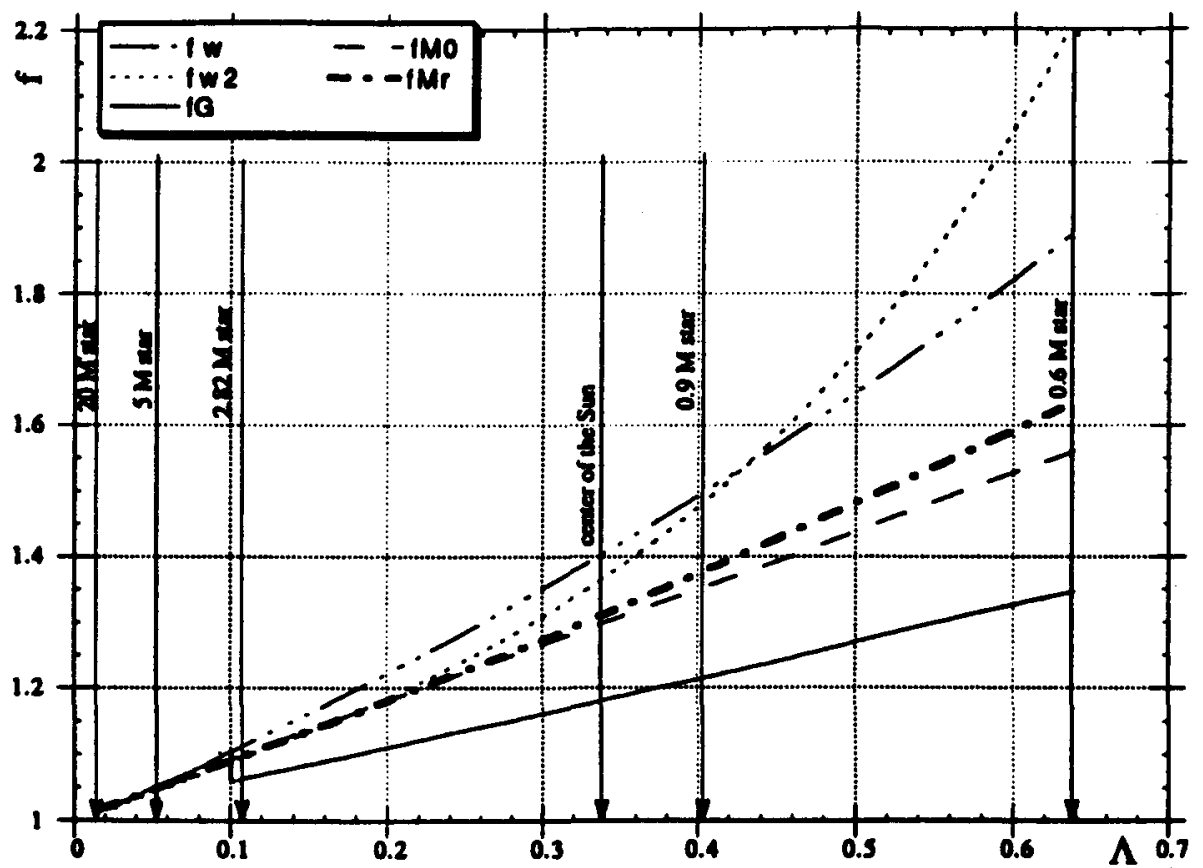

Fig. 40.1 Comparison of the analytic screening factors with precise values given by the $f_{M}$. radial dependent formalism for various ZAMS stars, and for the center of the present. Sun. The $p+{ }^{14} N$ reaction is here presented since the effects are larger than for the $p+p$ one for example.

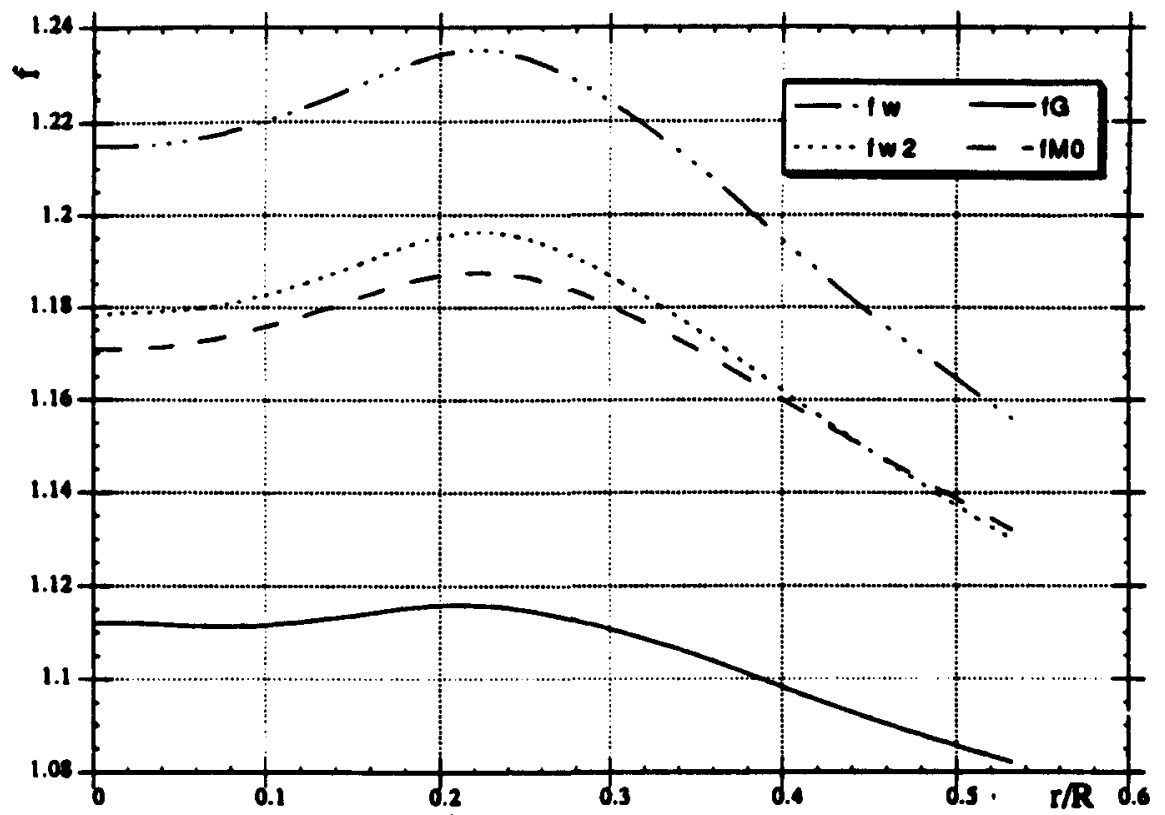

Fig. 40.2 f versus $r / R_{\odot)}$ for the ${ }^{7} B e(p, \gamma)^{8} B$ reaction. Note the $\approx 12 \%$ difference between $f_{i}$ and $f_{w}$ and the intermediate values given by $f_{M r}$.

with those calculated with $f_{M}$, for plasma conditions corresponding to the WS and IS regimes. The Figure above shows that all the prescriptions 
converge, as expected, toward the same value when $\Lambda$ is small enough. In low mass stars, high densities and low temperature increase the screening effect, and therefore the spread of these prescriptions is highlighted, but, in fact, in those cases, hydrogene burning is dominated by the $p p$ reaction. In the WS limit $(\Lambda<0.1)$, Mitler's prescription quite agrees with $f_{w 2}$ (a second order approximation in $\Lambda$ of the pair distribution functions involved in the WS calculation). The agreement between $f_{M 0}$ and $f_{M r}$ is very good in the solar case $(\approx 1.5 \%)$ while $f_{G}$ and $f_{w}$ respectively under and over estimate. $f_{M r}$ by about 10 and $8 \%$. The influence on the ${ }^{8} B$ neutrino predictions may be deduced from figure 1.2 where the screening factors are calculated for the ${ }^{7} B e(p, \gamma)^{8} B$ reaction. At the maximum rate of this reaction (which occurs at about $\left.\mathrm{r} / R_{\odot}=0.06\right) f_{w}$ and $f_{G}$ differ by $12 \%$, leading to the same difference on the chlorine and water predictions. This difference is smaller for the Gallium detectors $(\approx 3 \%)$, since about $60 \%$ of the predicted fluxes are due to $\mathrm{pp}$ neutrinos.

\subsection{Conclusion}

The $\mathrm{S} 54$ and GWGC prescriptions do not seem to be precise enough in the solar case, therefore, we recommend to use the simple $H(0)$ Mitler's formalism which avoids the discontinuities at $\Lambda=0.1$ and reproduces the radial dependent $H(r)$ prescription within $2 \%$ accuracy.

\section{References}

Turck-Chièze S.and Lopes I., Astrophys. J. 408, 34 (1993).

Turck-Chièze S., Däppen W., Fossat. E., Provost J., Schatzman E., Vignaud D., Phys. Rep. 230, 2-4, (1993)

Dzitko H. and Turck-Chieze S., in Advances in solar physics, ed. by (G. Belvedere and $\mathrm{M}$. Rodonò, in press

Salpeter E., Australian J. Phys. 7, 353, (1954)

Graboske et al, Astrophys. J. 181, 457, (1973)

Mitler H. E., Astrophys. J., 212, 513, (1977) 